\title{
Neutrophil-to-Lymphocyte Ratio (NLR) and Platelet- to-Lymphocyte Ratio (PLR) Are Potentially Valuable Tests for Diagnosing Periprosthetic Joint Infection
}

Hao Wu

Peking University First Hospital

Liping Pan

Peking University First Hospital

Zhichao Meng

Peking University First Hospital

Heng Liu

Peking University First Hospital

Xin Yang

Peking University First Hospital

Yongping Cao ( $\nabla$ freehorse66@163.com )

Peking University First Hospital https://orcid.org/0000-0003-0550-5991

\section{Research article}

Keywords: periprosthetic joint infection, diagnostic value, neutrophil-to-lymphocyte ratio, platelet-tolymphocyte ratio, platelet-to-mean platelet volume ratio

Posted Date: April 1st, 2021

DOI: https://doi.org/10.21203/rs.3.rs-376702/v1

License: (c) (1) This work is licensed under a Creative Commons Attribution 4.0 International License. Read Full License 


\section{Abstract \\ Background}

Diagnosis of periprosthetic joint infection (PJI) is challenging for clinicians, and the most commonly used methods are too complicated and expensive for many clinical practices. The neutrophil-to-lymphocyte ratio (NLR), platelet-to-lymphocyte ratio (PLR), and platelet-to-mean platelet volume ratio (PVR), are simple biomarkers of infection and can be easily determined from routine blood tests. The aim of our study is to determine the value of these three parameters for the diagnosis of PJI.

\section{Methods}

One hundred and sixty-four patients who received revision surgery after total knee or total hip replacements were enrolled, 47 in a PJI group and 117 in an aseptic failure group. Receiver operator characteristic (ROC) analysis was used to compare the performance of NLR, PLR, PVR, C reactive protein (CRP), and erythrocyte sedimentation rate (ESR) for the diagnosis of PJI.

\section{Results}

The levels of NLR, PLR, and PVR were significantly higher in patients with PJI (all P< 0.05$)$. ROC analysis showed that the area under curve (AUC) values of NLR (0.740), PLR (0.721), and PVR (0.668) were lower than those for CRP (0.896) and ESR (0.829). PLR had the highest sensitivity (85.11\%) among all tested biomarkers, and NLR improved the diagnostic performance when used with CRP.

\section{Conclusion}

NLR and PLR are potentially valuable tests for the diagnosis of PJI, but PVR only has limited diagnostic value.

\section{Introduction}

Periprosthetic joint infection (PJI) is a very serious complication that can occur after total knee arthroplasty (TKA) and total hip arthroplasty (THA) [1]. The incidence of PJI is about 0.5 to $2.5 \%$ after primary TKA or THA, and there are approximately 1.5 infections per 1000 person-joint years [2-5]. PJI has a negative impact on joint mobility and patient quality of life, causes significant morbidity, and accounts for substantial health care expenditures. The annual cost for revision operations following PJI in the US was $\$ 566$ million in 2009 and will increase to $\$ 1.62$ billion by 2020 [5]. However, surgeons still have difficulty in diagnosing PJI due to the lack of gold standard diagnostic criteria, and this can delay treatment and implementation of an appropriate treatment plan. 
The current Musculoskeletal Infection Society (MSIS) criteria are widely used for diagnosing PJI. These criteria consider the results from blood and synovial fluid tests, a clinical examination, intraoperative histology, and cultures [6]. Although use of these criteria significantly improves the accuracy of PJI diagnosis, many of the procedures are time-consuming, resource intensive, and invasive. Therefore, some clinicians have examined the use of inflammatory biomarkers for the timely diagnosis of PJI before revision operations. Miyamae et al. found the level of alpha-defensin in synovial fluid had high accuracy in diagnosing PJI, with a sensitivity of $93 \%$ and a specificity of $100 \%$ [7]. Other studies reported that synovial leukocyte esterase [8,9] and serum fibrinogen and D-Dimer [10-12] had value in the diagnosis of PJI. However, due to technical difficulties and the high cost required for measuring these parameters, primary hospitals do not commonly perform these measurements.

Recent studies considered the use of certain serum inflammatory biomarkers obtained from routine blood examinations for the diagnosis of PJI, due to their low-cost and ready availability in clinical practice. These include the mean platelet volume ratio (PVR), the neutrophil-to-lymphocyte ratio (NLR), and the platelet-to-lymphocyte ratio (PLR). For example, Paziuk et al. found that the PVR was associated with PJI (optimal cutoff: 31.70 ), and that PVR with C-reactive protein (CRP) and erythrocyte sedimentation rate (ESR) significantly improved the diagnostic accuracy of PJI compared with traditional biomarkers [13]. A recent study by Yu et al. showed that the NLR had a higher accuracy for early diagnosis of PJI than CRP and ESR, and their receiver operating characteristic (ROC) analysis indicated the area under curve (AUC) for NLR was 0.802 [14]. Tirumala et al. found that the PLR and PVR significantly improved the accuracy of PJI diagnosis when combined with traditional serum biomarkers and joint aspirate results, with sensitivity and specificity above $97 \%$ [15]. However, a recent study by Sigmund et al. had conflicting results, in that NLR (AUC: 0.68) and PVR (AUC: 0.62) had inferior performance in the diagnosis of PJI compared with traditional biomarkers. They therefore suggested these markers should not be used alone for the diagnosis of PJI [16].

Therefore, we designed the present single-center retrospective study to evaluate the performance of three biomarkers (NLR, PLR, and PVR) for the diagnosis of PJI and to compare their diagnostic performance with two traditional biomarkers, ESR and CRP.

\section{Materials And Methods}

This retrospective study was approved by Institutional Review Board (IRB) of Peking University 1st Hospital. The records of patients who received revision surgery after TKA or THA at our institution from January 2001 to December 2019 were reviewed from Electronic Medical Record System. According to the MSIS criteria for PJI diagnosis (Table 1), the enrolled patients were carefully reviewed and divided into two groups: a PJI group and an aseptic revision group [6]. Because it was difficult to determine the presence of persistent infections, patients who received second-stage reimplantation were excluded. The other exclusion criteria were: diagnosis of a periprosthetic fracture or joint dislocation; missing critical data (including ESR, CRP, NLR, PVR, and PLR); presence of an autoimmune disease, such as rheumatoid 
arthritis or ankylosing spondylitis; and history of a malignant tumor. Finally, 164 patients were enrolled, 47 in the PJI group and 117 in aseptic revision group (Fig. 1).

Table 1

According to MSIS criteria, PJI is diagnosed when a patient has one of the two major criteria or three of the five minor criteria.

\section{Major $\quad 1)$ Two positive periprosthetic cultures with phenotypically identical organisms Criteria \\ 2) A sinus tract communicating with the joint}

Minor 1) Elevated serum C-reactive protein (> $10 \mathrm{mg} / \mathrm{L})$ and erythrocyte sedimentation rate (> 30 Criteria $\mathrm{mm} / \mathrm{h}$ )

2) Elevated synovial fluid white blood cell count (>3000 cells $/ \mathrm{mL}$ ) or change on an leukocyte esterase test strip (+ or ++)

3) Elevated synovial fluid polymorphonuclear neutrophil percentage (>80\%)

4) Positive histological analysis of periprosthetic tissue ( $>5$ neutrophils/HPF) based on examination of 5 HPFs

5) A single positive culture

All venous blood samples were collected routinely by nurses on first or second day of admission and sent to the clinical laboratory of our hospital for testing. Joint aspiration for synovial fluid testing and the histological analysis of periprosthetic tissues was performed as needed. The periprosthetic tissues or synovial fluid collected intra-operatively were sent for aerobic and anaerobic cultures.

The basic information of patients, such as age, gender, body mass index (BMI), diagnosis, treatment, comorbidities, and the results of pre-operative serum biomarkers (ESR, CRP, NLR, PLR, PVR) were collected from the electronic medical records. Test results related to PJI diagnosis, such as those from joint aspirations, sinus tract, histological analysis, and pathogen culture findings, were also collected from these records.

\section{Statistical Analysis}

Quantitative data are expressed as means \pm standard deviations, and categorical data as frequencies and ratios. The independent samples $t$-test and the chi-squared test were used to compare the characteristics of the PJI and aseptic failure groups. Continuous variables were compared using the Mann-Whitney test and categorical variables using the chi-squared test. The diagnostic performances of the different biomarkers were compared based on the results of ROC analysis, with calculations of AUC, sensitivity, specificity, positive predictive value (PPV), and negative predictive value (NPV). The optimal threshold value for each biomarker was determined using the Youden $\mathrm{J}$ index. All statistical analyses were performed using MedCalc Statistical Software version 18.2.1 (Ostend, Belgium) and the figures were drawn using GraphPad Prism version 7 (San Diego, CA). The threshold for statistical significance was 0.05 for all analyses. 


\section{Results}

We retrospectively examined 164 patients who received revision surgery after TKA or THA, and used the MSIS criteria to classify 47 patients in PJI group and 117 patients in aseptic revision group (Table 2) [6]. The basic characteristics of each group were shown in Table-2. These two groups had no significant differences in $\mathrm{BMI}$ and gender, although the aseptic failure group was older $(P=0.013)$ and less likely to have TKA $(P<0.001)$. The PJI group had a greater CRP $(48.78 \pm 84.71$ vs. $4.25 \pm 5.61 \mathrm{mg} / \mathrm{L}, \mathrm{P}<0.001)$, ESR $(46.32 \pm 29.99 v s .14 .49 \pm 11.07 \mathrm{~mm} / \mathrm{h}, \mathrm{P}<0.001)$, NLR $(4.43 \pm 2.98 v s .2 .61 \pm 2.42, \mathrm{P}<0.001), \mathrm{PLR}$ $(220.84 \pm 126.95$ vs. $146.15 \pm 57.07, \mathrm{P}<0.001)$, and PVR $((34.59 \pm 12.72$ vs. $27.33 \pm 10.51, \mathrm{P}=0.001)$.

Table 2

Basic characteristics and biomarkers in the PJI and aseptic failure groups.

\begin{tabular}{|c|c|c|c|}
\hline Characteristic & $\begin{array}{l}\text { PJI } \\
(\mathrm{N}=47)\end{array}$ & $\begin{array}{l}\text { Aseptic Failure } \\
(\mathrm{N}=117)\end{array}$ & $P$ \\
\hline Age (years) & $63.55 \pm 11.56$ & $68.44 \pm 11.15$ & $0.013^{*}$ \\
\hline $\mathrm{BMI}\left(\mathrm{kg} / \mathrm{m}^{2}\right)$ & $24.74 \pm 3.44$ & $24.62 \pm 5.31$ & 0.887 \\
\hline Gender & & & 0.085 \\
\hline Male & 22 & 38 & \\
\hline Female & 25 & 79 & \\
\hline Affected Joint & & & $<0.001 *$ \\
\hline Knee & 28 & 21 & \\
\hline Hip & 19 & 96 & \\
\hline \multicolumn{4}{|l|}{ Biomarkers } \\
\hline CRP (mg/L) & $48.78 \pm 84.71$ & $4.25 \pm 5.61$ & $<0.001 *$ \\
\hline $\operatorname{ESR}(\mathrm{mm} / \mathrm{h})$ & $46.32 \pm 29.99$ & $14.49 \pm 11.07$ & $<0.001 *$ \\
\hline NLR & $4.43 \pm 2.98$ & $2.61 \pm 2.42$ & $<0.001 *$ \\
\hline PLR & $220.84 \pm 126.95$ & $146.15 \pm 57.07$ & $<0.001 *$ \\
\hline PVR & $34.59 \pm 12.72$ & $27.33 \pm 10.51$ & $0.001 *$ \\
\hline $\begin{array}{l}\text { Values are give } \\
\text { BMI: Body mas } \\
\text { PLR: Platelet to } \\
\text { PVR: Platelet to }\end{array}$ & $\begin{array}{l}\text { index; LR: Neutrop } \\
\text { ymphocyte ratio; } \\
\text { nean platelet volu }\end{array}$ & $\begin{array}{l}\text { il to lymphocyte } \\
\text { e ratio; }\end{array}$ & tio; \\
\hline
\end{tabular}


ROC analyses (Fig. 2 and Table 3) indicated that CRP performed best in diagnosing PJI (AUC: $0.896,95 \%$ Cl: 0.838-0.938; optimal cutoff: $6.59 \mathrm{mg} / \mathrm{L}$ ), followed by ESR (AUC: $0.829,95 \%$ Cl: $0.763-0.883$; optimal cutoff: $34 \mathrm{~mm} / \mathrm{h}$ ). The diagnostic performance of NLR (AUC: $0.740,95 \% \mathrm{Cl}$ : $0.666-0.805$; optimal cutoff: 2.71) and PLR (AUC: $0.721,95 \% \mathrm{Cl}$ : 0.646-0.788; optimal cutoff: 132.67) were fair, and the diagnostic performance of PVR (AUC: 0.668; 95\% Cl: 0.590-0.739; optimal cutoff: 34.31) was poor.

\begin{tabular}{|c|c|c|c|c|c|c|}
\hline Biomarker & AUC (95\%Cl) & $\begin{array}{l}\text { Optimal cut- } \\
\text { off }\end{array}$ & Sensitivity & Specificity & PPV & NPV \\
\hline CRP & $\begin{array}{l}0.896(0.838- \\
0.938)\end{array}$ & $6.59 \mathrm{mg} / \mathrm{L}$ & $82.98 \%$ & $83.76 \%$ & $67.24 \%$ & $92.45 \%$ \\
\hline ESR & $\begin{array}{l}0.829(0.763- \\
0.883)\end{array}$ & $34 \mathrm{~mm} / \mathrm{h}$ & $63.83 \%$ & $95.73 \%$ & $85.72 \%$ & $86.82 \%$ \\
\hline NLR & $\begin{array}{l}0.740(0.666- \\
0.805)\end{array}$ & 2.71 & $68.09 \%$ & $70.09 \%$ & $47.77 \%$ & $84.54 \%$ \\
\hline PLR & $\begin{array}{l}0.721(0.646- \\
0.788)\end{array}$ & 132.67 & $85.11 \%$ & $51.28 \%$ & $41.24 \%$ & $89.55 \%$ \\
\hline PVR & $\begin{array}{l}0.668(0.590- \\
0.739)\end{array}$ & 34.31 & $46.81 \%$ & $83.76 \%$ & $53.66 \%$ & $79.68 \%$ \\
\hline
\end{tabular}

As shown in Table-3, at the cut-off value of 2.71 for NLR, the sensitivity, specificity, positive predictive value (PPV), and negative predictive value (NPV) was $68.09 \%, 70.09 \%, 47.77 \%$, and $84.54 \%$, respectively. The optimal threshold for PLR was 132.67 with sensitivity $85.11 \%$, specificity $51.28 \%$, PPV $41.24 \%$, and NPV $89.55 \%$. The optimal cut-off for PVR was 34.31 showing sensitivity $46.81 \%$, specificity $83.76 \%$, PPV $53.66 \%$, and NPV $79.68 \%$. The optimal predictive cut-off value for ESR and CRP was $34 \mathrm{~mm} / \mathrm{h}$ (sensitivity $63.83 \%$, specificity $95.73 \%$, PPV $85.72 \%$, and NPV $86.82 \%$ ) and $6.59 \mathrm{mg} / \mathrm{L}$ (sensitivity $82.98 \%$, specificity 83.76\%, PPV 67.24\%, and NPV 92.45\%).

We performed pair-wise comparisons of AUC values to assess the diagnostic performance of the different biomarkers (Table 4). CRP was significantly better than NLR $(P=0.0003), P L R(P=0.0001)$, and PVR $(P<0.0001)$, but not significantly different from ESR $(P=0.0711)$. ESR was significantly better than PLR $(P=0.0466)$ and PVR $(P=0.0035)$, but not significantly different from NLR $(P=0.0747)$. There were no significant differences of NLR, PLR, and PVR. 
Table 4. Pair-wise comparisons of AUC values from use of different individual biomarkers for the diagnosis of PJI.

\begin{tabular}{|llllll|} 
& CRP & ESR & NLR & PLR & PVR \\
\hline CRP & - & - & - & - & - \\
\hline ESR & 0.071 & - & - & - & - \\
\hline NLR & $<0.001^{*}$ & 0.075 & - & - & - \\
\hline PLR & $<0.001 *$ & $0.047^{*}$ & 0.669 & - & - \\
PVR & $<0.001 *$ & $0.004^{*}$ & 0.259 & 0.199 & \\
$* P<0.05$ & & & & & \\
\hline
\end{tabular}

We also determined the diagnostic performance of CRP combined with each of the four other biomarkers (Table 5). The resulting AUC values indicated that each combination had a very good diagnostic performance, with CRP + PVR having the lowest AUC (0.885, 95\% Cl: 0.825-0.929) and CRP + NLR having the highest AUC $(0.897,95 \% \mathrm{Cl}: 0.840-0.939)$. However, none of these combinations was significantly better than CRP alone (all $\mathrm{P}>0.423$ ).

Table 5. Performance of the combined use of CRP with other biomarkers for the diagnosis of PJI.

$\begin{array}{llll}C R P+E S R & C R P+N L R & C R P+P L R & C R P+P V R\end{array}$

$\begin{array}{lllll}\text { AUC } & 0.890(0.832- & 0.897(0.840- & 0.888(0.830- & 0.885(0.825- \\ (95 \% \mathrm{Cl}) & 0.934) & 0.939) & 0.932) & 0.929)\end{array}$

\begin{tabular}{lllll} 
Sensitivity & $74.47 \%$ & $82.98 \%$ & $85.11 \%$ & $68.09 \%$ \\
\hline Specificity & $94.02 \%$ & $83.76 \%$ & $77.78 \%$ & $90.60 \%$ \\
\hline PPV & $83.34 \%$ & $67.24 \%$ & $60.61 \%$ & $74.42 \%$ \\
\hline NPV & $90.16 \%$ & $92.45 \%$ & $92.86 \%$ & $87.61 \%$ \\
\hline P value & 0.791 & 0.423 & 0.479 & 0.519
\end{tabular}

(vs. CRP)

AUC: area under the curve; PPV: positive predictive value; NPV: negative predictive value. 


\section{Discussion}

PJI is a very serious complication that can occur after total joint arthroplasty, which leads to a devastating consequence if not diagnosed properly. Thus, the MSIS initially proposed criteria for diagnosis of PJI, and modified these criteria during the International Consensus Meeting (ICM) in 2013 [6]. However, it is still difficult to diagnose PJI pre-operatively because synovial fluid can be difficult to obtain via joint aspiration and bacterial cultures may take a long time to yield results. In an effort to accurately diagnose PJI in a more timely manner, several previous studies examined the potential use of novel biomarkers such as CD $4^{+}$blood monocytes [17], a-defensin [18], leukocyte esterase [8], and calprotectin [19] and found that they had greater diagnostic value than traditional biomarkers, including CRP and ESR. But, measurement of these novel parameters can be expensive and unavailable in some institutions. Our purpose was to identify simple and practical biomarkers for the early diagnosis of PJI. Thus, we assessed the diagnostic performance of NLR, PLR, and PVR, the biomarkers that are easily obtained from routine blood tests, and then compared their diagnostic performance with the traditional biomarkers.

We first compared the diagnostic performance of NLR, PLR, PVR in distinguishing patients in the PJI and the aseptic failure groups. Each of these three biomarkers was significantly elevated in PJI patients compared with aseptic failure patients, similar to the traditional biomarkers. Our ROC analysis indicated the optimal cut-off values were 2.71 for NLR, 132.67 for PLR, and 34.31 for PVR. NLR had the best diagnostic performance (AUC: $0.740,95 \% \mathrm{Cl}: 0.666-0.805$ ), with a sensitivity of $68.09 \%$ and a specificity of $70.09 \%$, followed by PLR (AUC: $0.721,95 \% \mathrm{Cl}: 0.646-0.788$ ) and PVR (AUC: $0.668,95 \%$ Cl: $0.590-$ 0.739). Notably, PLR had the highest sensitivity (85.11\%) among all five tested biomarkers, and its sensitivity was even greater than that of CRP (82.98\%) and ESR (63.83\%). However, CRP (AUC: 0.896) and ESR (AUC: 0.829) provided better overall diagnostic performance than NLR, PLR, and PVR. Thus, we also tested the diagnostic performance of the combined use of CRP with each of the other markers. The combination of CRP + NLR had a diagnostic performance similar to that of CRP + ESR, the combination widely used in clinical practice.

Besides the convenience and minimal expense necessary for measuring the three biomarkers examined here, previous studies reported that these three biomarkers are generally useful for the diagnosis of infection. For example, Zareifar et al. [20] reported that an elevated platelet count and mean platelet volume (MPV) were decreased in patients with infectious and inflammatory processes. A retrospective study by Paziuk et al. [13] demonstrated that the PVR had acceptable performance in diagnosing PJI, with an AUC of 0.69 ( $48.10 \%$ sensitivity, $80.85 \%$ specificity) at the cut-off value of 31.70 , similar to our cutoff value (34.31). They also reported that the combined application of PVR, CRP, and ESR significantly improved diagnostic performance. Sigmund et al. [16] evaluated the diagnostic value of PVR in a cohort of 177 patients who required revision surgery after THA $(n=91)$ and TKA $(n=86)$. They found that the individual use of PVR only had a sensitivity of $43 \%$ and a specificity of $81 \%$, significantly inferior to that of CRP. They also found that the combined use of CRP + PVR did not improve the diagnostic performance 
relative to CRP alone. Our results are consistent with those of Sigmund et al [16]. We therefore conclude that PVR should not be considered as a reliable test for the diagnosis of PJI.

Other studies also considered the use of NLR and PLR as biomarkers for inflammatory responses and infections. For example, Qu et al. [21] measured the NLR in 2160 patients with bloodstream infections (BSIs) and 2523 healthy controls and found that the NLR was significantly higher in the BSI group. They concluded that NLR had a strong association with BSIs caused by Gram-negative bacteria, Gram-positive bacteria, and fungi. Shen et al. [22] demonstrated that an elevated PLR was related to increased risk of mortality based on analysis of the clinical data of 5537 patients with sepsis. Some other recent studies found that NLR and PLR had potential use for predicting PJI. In particular, the retrospective study by Zhao et al. [23] demonstrated that the NLR and PLR were significantly higher in an early PJI group than in a non-PJI group, and that NLR might be more valuable than PLR based on ROC analysis. They used an NLR cutoff of 2.77, similar to our cutoff (2.71). Similarly, Yu et al. [14] found that the NLR was effective in diagnosing PJI (AUC: 0.802 , 85\% sensitivity, 68.3\% specificity, 34.7\% PPV, 95.8\% NPV) with a cut-off value of 2.13. However, Zhao et al. [23] and Yu et al. [14] demonstrated that NLR and PLR had greater predictive value for diagnosis of PJI than ESR and CRP, in stark contrast to our results. Our further analysis indicated that this was most likely due to differences in the characteristics of the enrolled patients; in our study, examined patients were diagnosed as chronic PJI, but the other two studies $[14,23]$ examined patients who had acute PJI.

To improve the accuracy of PJI diagnosis, many researchers have examined the effect of the combined use of several biomarkers. In particular, Tirumala et al. [15] found that the sensitivity and specificity for diagnosing PJI was near-perfect when PLR or PVR was combined with CRP, ESR, and synovial biomarkers. The present study showed that that only CRP + NLR provided better diagnostic performance than CRP + ESR (the most widely used combination in clinical practice) for PJI diagnosis, and that the difference was not statistically significant. Therefore, we consider NLR to be potentially valuable for the diagnosis of PJI.

There are several limitations in our study. First, this is a retrospective study and therefore has all of the limitations inherent to studies with this design. Our exclusion of patients with missing critical data or complicated with autoimmune diseases might have led to some bias. Second, there is no gold standard for the diagnosis of PJI. However, the MSIS criteria are considered the best method for its diagnosis, although this standard has low sensitivity in patients with low-virulence bacterial infections [17, 24]. To reduce the possibility of misdiagnosis, we excluded patients who underwent second-stage reimplantation due to the difficulty of determining their infection status. Finally, we examined 164 cases from a single institution, thus the small sample limited the generalizability of our conclusions. Therefore, well-designed multicenter studies with larger samples are needed to evaluate the value of NLR, PLR, and PVR for the diagnosis of PJI.

\section{Conclusion}


In conclusion, we found that the levels of NLR, PLR, and PVR were significantly higher in patients diagnosed with PJI, and these biomarkers therefore may have potential for the diagnosis of PJI. However, when used alone, each of these biomarkers only had fair diagnostic performance compared with CRP and ESR. However, our analysis also showed that PLR had the highest sensitivity among all five tested biomarkers, and that NLR improved the diagnostic performance when used in conjunction with CRP. Therefore, we conclude that NLR and PLR are potentially valuable biomarkers for diagnosing PJI, but PVR has only limited diagnostic value.

\section{Abbreviations}

NLR: neutrophil-to-lymphocyte ratio; PLR: platelet-to-Lymphocyte ratio; PVR: platelet-to-mean platelet volume ratio; CRP: C reactive protein; ESR: erythrocyte sedimentation rate; MPV: mean platelet volume; PJI: periprosthetic joint infection; ROC: receiver operator characteristic; AUC: area under curve; TKA: total knee arthroplasty; THA: total hip arthroplasty; MSIS: musculoskeletal infection society; BMI: body mass index; PPV: positive predictive value; NPV: negative predictive value; BSI: bloodstream infections;

\section{Declarations}

\section{Ethics approval and consent to participate}

The study was approved by Institutional Review Board (IRB) of Peking University 1st Hospital.

\section{Consent for publication}

Not applicable.

\section{Availability of data and materials}

According to the policy of our hospital, the raw data could not be shared with others without permission. An anonymised form of the data could be made available from the corresponding author upon reasonable request.

\section{Competing interests}

The authors declare that they have no competing interests.

\section{Funding}

No funding

\section{Authors' contributions}

Study design: Hao Wu, Liping Pan, Yongping Cao. Data collection and nalysis: Hao Wu, Zhichao Meng. Paper writing: Hao Wu, Liping Pan; Critical revision of the paper: Yongping Cao, Xin Yang, Heng Liu. All 
authors read and approved the final version of the manuscript

\section{Acknowledgments}

The authors would like to thank all colleagues from the Department of Orthopedics, Peking University First Hospital, for the help and advices in the process of data collection, results analysis and paper writing.

\section{References}

1. Tetreault MW, Wetters NG, Aggarwal V, et al. The Chitranjan Ranawat Award: Should prophylactic antibiotics be withheld before revision surgery to obtain appropriate cultures? Clin Orthop Relat Res. 2014;472:52-6. DOl:10.1007/s11999-013-3016-5.

2. Huotari K, Peltola M, Jamsen $E$. The incidence of late prosthetic joint infections: a registry-based study of 112,708 primary hip and knee replacements. Acta Orthop. 2015;86:321-5. DOI:10.3109/17453674.2015.1035173.

3. Gundtoft PH, Overgaard S, Schonheyder HC, et al. The "true" incidence of surgically treated deep prosthetic joint infection after 32,896 primary total hip arthroplasties: a prospective cohort study. Acta Orthop. 2015;86:326-34. DOI:10.3109/17453674.2015.1011983.

4. Beam E, Osmon D. Prosthetic Joint Infection Update. Infect Dis Clin N Am. 2018;32:843-59. DOI:10.1016/j.idc.2018.06.005.

5. Kurtz SM, Lau E, Watson $\mathrm{H}$, et al. Economic burden of periprosthetic joint infection in the United States. J Arthroplast. 2012;27(e61):61-5. DOI:10.1016/j.arth.2012.02.022.

6. Parvizi J, Gehrke T, International Consensus Group on Periprosthetic Joint I. Definition of periprosthetic joint infection. J Arthroplast. 2014;29:1331. DOI:10.1016/j.arth.2014.03.009.

7. Miyamae Y, George J, Klika AK, et al. Diagnostic Accuracy of the Alpha-Defensin Test for Periprosthetic Joint Infection in Patients With Inflammatory Diseases. J Arthroplast. 2019;34:176771. DOI:10.1016/j.arth.2019.04.020.

8. Tischler EH, Cavanaugh PK, Parvizi J. Leukocyte esterase strip test: matched for musculoskeletal infection society criteria. The Journal of bone joint surgery American volume. 2014;96:1917-20. DOI:10.2106/JBJS.M.01591.

9. Shahi A, Alvand A, Ghanem E, et al. The Leukocyte Esterase Test for Periprosthetic Joint Infection Is Not Affected by Prior Antibiotic Administration. The Journal of bone joint surgery American volume. 2019;101:739-44. DOI:10.2106/JBJS.18.00615.

10. Shahi A, Kheir MM, Tarabichi M, et al. Serum D-Dimer Test Is Promising for the Diagnosis of Periprosthetic Joint Infection and Timing of Reimplantation. The Journal of bone joint surgery American volume. 2017;99:1419-27. DOI:10.2106/JBJS.16.01395.

11. Wu H, Meng Z, Pan L, et al. Plasma Fibrinogen Performs Better Than Plasma d-Dimer and Fibrin Degradation Product in the Diagnosis of Periprosthetic Joint Infection and Determination of 
Reimplantation Timing. J Arthroplast. 2020;35:2230-6. DOI:10.1016/j.arth.2020.03.055.

12. Li R, Shao H-Y, Hao L-B, et al. Plasma Fibrinogen Exhibits Better Performance Than Plasma D-Dimer in the Diagnosis of Periprosthetic Joint Infection. J Bone Joint Surg. 2019;101:613-9. DOI:10.2106/jbjs.18.00624.

13. Paziuk T, Rondon AJ, Goswami K, et al. A Novel Adjunct Indicator of Periprosthetic Joint Infection: Platelet Count and Mean Platelet Volume. J Arthroplast. 2020;35:836-9. DOI:10.1016/j.arth.2019.10.012.

14. Yu BZ, Fu J, Chai W, et al. Neutrophil to lymphocyte ratio as a predictor for diagnosis of early Periprosthetic joint infection. BMC Musculoskelet Disord. 2020;21:706. DOI:10.1186/s12891-02003704-5.

15. Tirumala V, Klemt C, Xiong L, et al. Diagnostic Utility of Platelet Count/Lymphocyte Count Ratio and Platelet Count/Mean Platelet Volume Ratio in Periprosthetic Joint Infection Following Total Knee Arthroplasty. J Arthroplast. 2021;36:291-7. DOI:10.1016/j.arth.2020.07.038.

16. Sigmund IK, Holinka J, Staats K, et al. Inferior performance of established and novel serum inflammatory markers in diagnosing periprosthetic joint infections. International orthopaedics. 2020. DOI:10.1007/s00264-020-04889-z.

17. Qu PF, Li R, Xu C, et al. A Clinical Pilot Study to Evaluate CD64 Expression on Blood Monocytes as an Indicator of Periprosthetic Joint Infection. The Journal of bone joint surgery American volume. 2020;102:e99. DOI:10.2106/JBJS.20.00057.

18. Sharma K, Ivy M, Block DR, et al. Comparative analysis of 23 synovial fluid biomarkers for hip and knee periprosthetic joint infection detection. J Orthop Res. 2020;38:2664-74. DOI:10.1002/jor.24766.

19. Salari P, Grassi M, Cinti B, et al. Synovial Fluid Calprotectin for the Preoperative Diagnosis of Chronic Periprosthetic Joint Infection. J Arthroplast. 2020;35:534-7. DOI:10.1016/j.arth.2019.08.052.

20. Zareifar S, Farahmand Far MR, Golfeshan F, et al. Changes in Platelet Count and Mean Platelet Volume During Infectious and Inflammatory Disease and Their Correlation With ESR and CRP. J Clin Lab Anal. 2014;28:245-8. DOI:10.1002/jcla.21673.

21. Qu J, Yuan HY, Huang Y, et al. Evaluation of neutrophil-lymphocyte ratio in predicting bloodstream infection. Biomark Med. 2019;13:1255-61. DOI:10.2217/bmm-2018-0253.

22. Shen $Y$, Huang $X$, Zhang W. Platelet-to-lymphocyte ratio as a prognostic predictor of mortality for sepsis: interaction effect with disease severity-a retrospective study. BMJ open. 2019;9:e022896. DOI:10.1136/bmjopen-2018-022896.

23. Zhao G, Chen J, Wang J, et al. Predictive values of the postoperative neutrophil-to-lymphocyte ratio, platelet-to-lymphocyte ratio, and lymphocyte-to-monocyte ratio for the diagnosis of early periprosthetic joint infections: a preliminary study. J Orthop Surg Res. 2020;15:571. DOI:10.1186/s13018-020-02107-5.

24. Renz N, Yermak K, Perka C, et al. Alpha Defensin Lateral Flow Test for Diagnosis of Periprosthetic Joint Infection: Not a Screening but a Confirmatory Test. The Journal of bone joint surgery American volume. 2018;100:742-50. DOI:10.2106/JBJS.17.01005. 
Figures

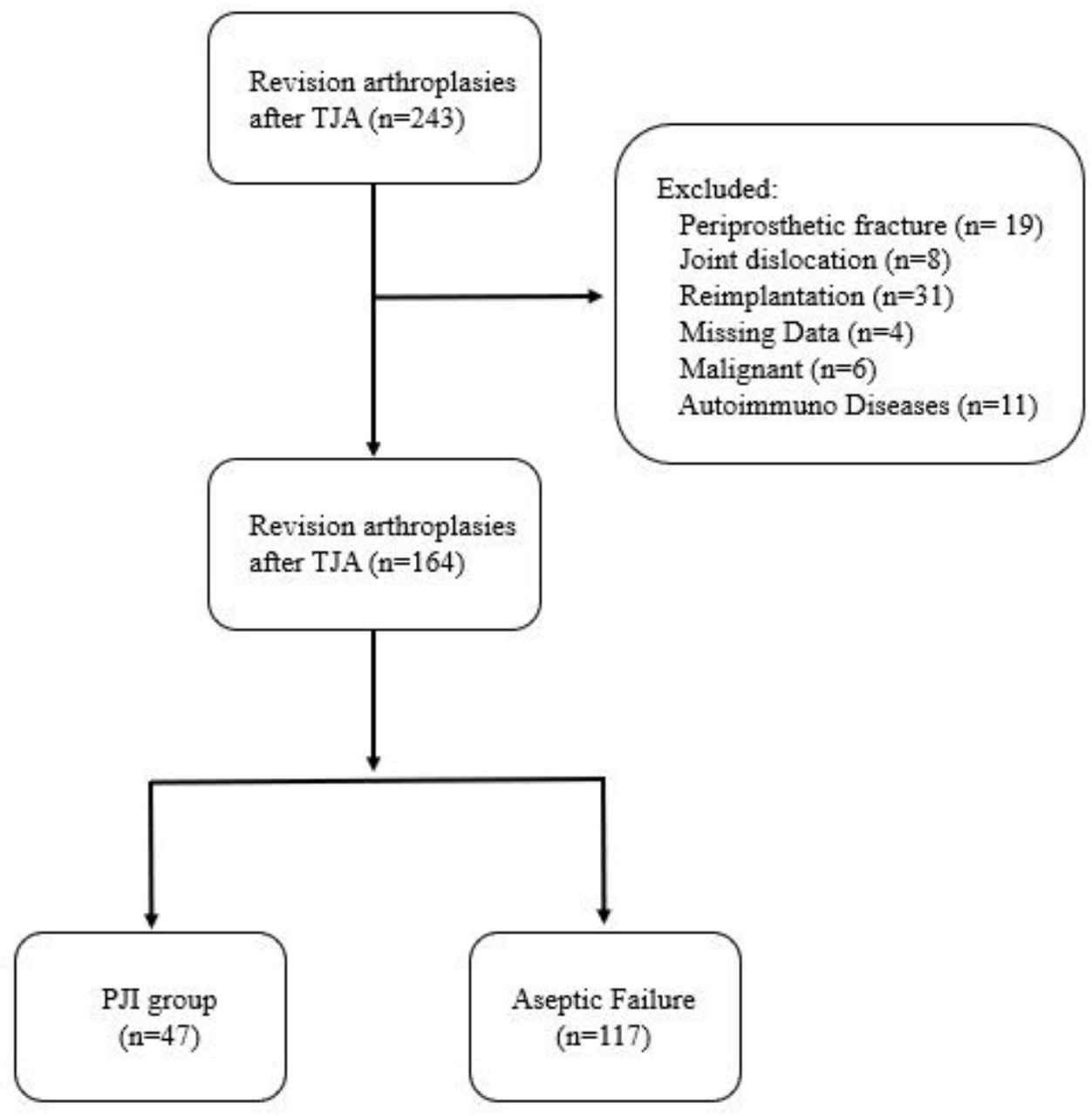

\section{Figure 1}

Disposition of patients who required revision arthroplasty after TKA or THA. 


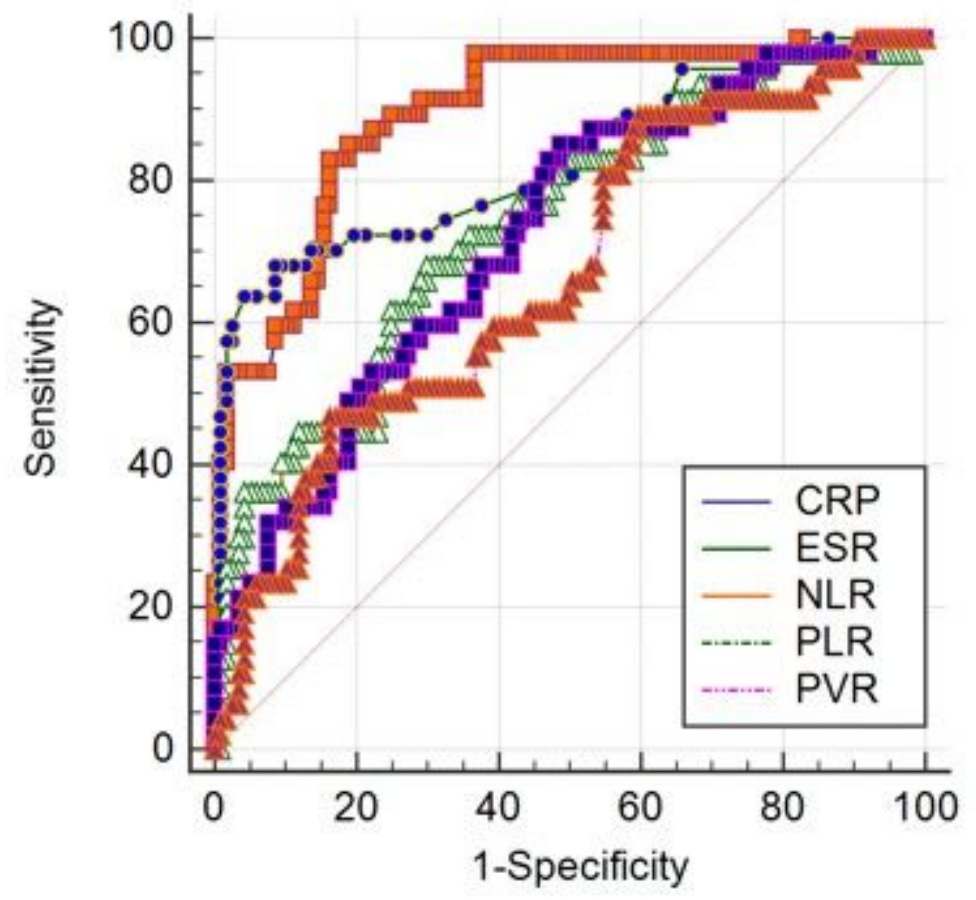

Figure 2

ROC curves for the diagnosis of PIJ based on NLR, PLR, and PVR relative to CRP and ESR (traditional biomarkers). 